\title{
Single Molecule Localization Microscopy at Janelia's Advanced Imaging Center
}

Jesse Aaron

HHMI Janelia Research Campus, Ashburn, Virginia, United States

Single molecule localization microscopy (SMLM) was initially demonstrated nearly 15 years ago in a trio of seminal papers. Since then, it has been recognized an integral method among the broader superresolution imaging repertoire available to biologists. As such, a variety of $2 \mathrm{D}$ and $3 \mathrm{D}$ commercial implementations are currently available and in use. However, there continues to be obstacles to its wider dissemination and utilization - particularly in a open-access setting. Fundamentally, as with nearly any other optical microscopy technique, commercial SMLM typically suffers from poorer axial vs. lateral resolution. This can hamper some biological queries. More broadly, the technical requirements for sample preparation, image acquisition, as well as processing and data analysis can be substantial for many bioscience researchers.

The Advanced Imaging Center (AIC) at Janelia Research Campus offers a novel access model to a precommercial implementation of SMLM via the interferometric photoactivation and localization microscope (iPALM), originally developed by Harald Hess and colleagues [1]. In contrast to other SMLM methods, this instrument achieves axial resolutions that meet or exceed the lateral values. This feature makes it uniquely suited to answer a suite of biological questions. To illustrate this point, representative example studies conducted at the AIC will be highlighted. These include measuring protein conformational changes [2] and clustering behavior [3], as well as characterizing pluripotent stem cell focal adhesion architecture [4], determining HIV particle assembly [5] behaviors, and the structure of a novel cell motility inhibitory factor [6]. In each case, novel observations were made possible only by the unique resolving capabilities of this class of microscopy.

While these examples serve to showcase some unique applications of the iPALM system, they also illustrate the challenges in making such technology broadly available. The AIC is tasked to not only offer free access to advanced pre-commercial imaging technologies, but also to guide and support users in all aspects of their project - from initial hypothesis creation, to sample preparation and experimental protocol validation, to image acquisition, through data processing and analysis. Therefore, successfully implementing open-access SMLM imaging depends strongly on a coordinated network of infrastructure and expertise. Careful collaboration between instrument developers, cell/tissue culture facilities, and fluorescent labeling tool builders, together with applications scientists and AIC visitors is essential. Furthermore, the task of data storage, processing, and analysis is formidable. Therefore, further discussion will be given as to how the AIC also aids users in translating unique data into meaningful biological insights.

\section{References}

[1] Shtengel, Gleb, James A. Galbraith, Catherine G. Galbraith, Jennifer Lippincott-Schwartz, Jennifer M. Gillette, Suliana Manley, Rachid Sougrat et al. "Interferometric fluorescent super-resolution microscopy resolves 3D cellular ultrastructure." Proceedings of the National Academy of Sciences 106, no. 9 (2009): 3125-3130.

[2] Moore, T.I., Aaron, J., Chew, T.L., Springer, T.A. (2018). Measuring integrin conformational change on the cell surface with super-resolution microscopy. Cell Reports, 22 (7). 1903-12.

[3] Griffié, J., Shlomovich, L., Williamson, D. J., Shannon, M., Aaron, J., Khuon, S., Burn, G. L., Boelen, L., Peters, R., Cope, A. P., Cohen, E. A. K., Rubin-Delanchy, P., Owen, D. M. (2017). 3D Bayesian cluster 
analysis of super-resolution data reveals LAT recruitment to the T cell synapse. Scientific Reports, 7(1), $1-9$.

[4] Stubb, Aki, Camilo Guzmán, Elisa Närvä, Jesse Aaron, Teng-Leong Chew, Markku Saari, Mitro Miihkinen, Guillaume Jacquemet, and Johanna Ivaska. "Superresolution architecture of cornerstone focal adhesions in human pluripotent stem cells." Nature communications 10, no. 1 (2019): 1-15.

[5] Buttler, C.A., Pezeshkian, N., Fernandez, M.V., Aaron, J.S., Norman, S., Freed, E.O., van Engenlenburg, S.B. (2018). Single molecule fate of HIV-1 Envelope reveals late-stage viral lattice incorporation. Nat. Comm. 9(1) 1861-76.

[6] Padilla-Rodriguez, M., Parker, S.S., Adams, D.G., Westerling, T., Puleo, J.I., Watson, A.W., Hill, S.M., Noon, M., Gaudin, R., Aaron, J., Tong, D., Roe, D.J., Knudsen, B., Mouneimne, G. (2018) The actin cytoskeletal architecture of estrogen receptor positive breast cancer cells suppresses invasion. Nat. Comm. 9(2980) 1-16. 